\title{
Awareness and Availability of Routine Germline BRCA1/2 Mutation Testing in Patients with Advanced Breast Cancer in Germany
}

\author{
Michael P. Lux ${ }^{a, b, c}$ Thomas Decker ${ }^{d}$ Eva Diana Runkel ${ }^{e}$ Alexander Niyazov $^{f}$ \\ Ruben G.W. Quek ${ }^{g}$ Norbert Marschner ${ }^{\text {h }}$ Nadia Harbeck ${ }^{i}$

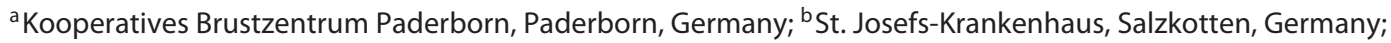 \\ 'Frauen- und Kinderklinik St. Louise, Paderborn, Germany; ${ }^{\mathrm{d} O n k o l o g i e ~ R a v e n s b u r g, ~ R a v e n s b u r g, ~ G e r m a n y ; ~}$

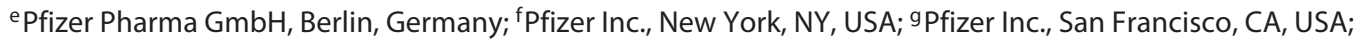 \\ hiOMEDICO AG, Freiburg, Germany; 'Brustzentrum, Frauenklinik und CCCLMU, LMU Klinikum, Universität München \\ (LMU), Munich, Germany
}

\section{Keywords}

$B R C A 1 / 2 \cdot$ Mutation testing · Germany $\cdot$ Advanced breast cancer · PARP inhibitors

\begin{abstract}
Introduction: Diagnostic testing of germline mutations in breast cancer susceptibility genes 1 or 2 ( $\mathrm{g} B R C A 1 / 2)$ in patients with human epidermal growth factor receptor 2 negative (HER2-) advanced breast cancer (ABC; locally advanced or metastatic breast cancer) is necessary to assess eligibility for poly(ADP-ribose) polymerase inhibitors (PARPi). We investigated awareness, clinical practice, and the availability of gBRCA1/2 mutation testing in the German outpatient oncology setting. Methods: Office-based oncologists completed a 23-item online survey. Responses were evaluated collectively and by center type. Results: Of 50 oncologists, 33 and 17 were medical and gynecological oncologists, respectively. Oncologists treated a median of 65 (range 14-350) patients with $A B C$ per year. The strongest decision factors to initiate gBRCA1/2 mutation testing were: patient's known family history of gBRCA1/2 mutation-related cancer(s), guideline recommendations, and triple-negative breast cancer (TNBC). In routine practice, $86 \%$ of oncologists tested for gBRCA1/2 mutations. Most oncologists (76-98\%) reported testing patients with a known family history of gBRCA1/2 mutation-related cancer(s) irrespective of receptor status. For unknown
\end{abstract}

family history, $92 \%$ of oncologists reported testing patients with advanced TNBC versus $30 \%$ for $\mathrm{HR}+/ \mathrm{HER} 2-\mathrm{ABC}$. Oncologists $(66 \%)$ rated the awareness of therapeutic relevance of gBRCA1/2 mutation testing for targeted treatment selection as good to satisfactory; $22 \%$ rated awareness as poor to insufficient. Conclusion: Diagnostic gBRCA1/2 mutation testing in patients with HER2-ABC is available and routinely performed in Germany's outpatient oncology setting. However, specific patient subgroups were not routinely tested despite therapeutic indications. Given PARPi availability, opportunities exist to improve testing rates especially for patients with $\mathrm{HR}+/ \mathrm{HER} 2-\mathrm{ABC}$ without a known family history of $\mathrm{g} B R C A 1 / 2$ mutation-related cancer(s).

(c) 2021 The Author(s)

Published by S. Karger AG, Basel

\section{Introduction}

Germline mutations in breast cancer susceptibility genes 1 or $2(\mathrm{gBRCA1/2})$ are associated with approximately $20-25 \%$ of hereditary breast cancers [1-4] and found in approximately $5-10 \%$ of all patients with breast cancer [1]. Individuals carrying a gBRCA $1 / 2$ mutation have an increased lifetime risk for development of certain cancers, including breast cancer $[5,6]$, and are commonly up to 25

R.G.W.Q. was an employee of Pfizer at the time of the analyses. mercial purposes requires written permission. 
years younger at diagnosis than the total breast cancer population [7]. Predictive testing in individuals with a familial predisposition to a known or likely pathogenic $\mathrm{gBRCA} 1 / 2$ mutation is well established in Western countries [6]. Identified carriers of gBRCA1/2 mutations also have access to intensified surveillance programs to ensure a prompt diagnosis if cancer develops [8].

The poly(ADP-ribose) polymerase inhibitors (PARPi) talazoparib and olaparib are the first approved targeted treatment options specifically for adult patients with human epidermal growth factor receptor 2 negative (HER2-) advanced breast cancer (ABC) and a gBRCA1/2 mutation [9-12]. Olaparib and talazoparib are available and have been reimbursed in Germany since July 2019 and June 2020 , respectively $[13,14]$. The pivotal randomized phase 3 studies EMBRACA [15] and OlympiAD [16] compared talazoparib and olaparib, respectively, with a single-agent chemotherapy of the physician's choice (eribulin, vinorelbine, capecitabine, or as a fourth option in EMBRACA only, gemcitabine). In both studies, treatment significantly improved progression-free survival and improved objective response rates, but not overall survival in the full intent-to-treat population [15-19]. Both PARPi exhibited a manageable safety profile, and significantly improved patient-reported quality-of-life versus chemotherapy [15, 16, 20-22]. Based on clinical evidence, PARPi are recommended treatments for patients with HER2- ABC and proven gBRCA1/2 mutations [23-25].

Consequently, international and national evidencebased consensus guidelines extended their recommendation for early and broad diagnostic gBRCA1/2 testing when therapeutically relevant [23-26]; NCCN broadened their guidelines to test any breast cancer subtype associated with a gBRCA1/2 mutation [24]. Despite these recommendations, testing rates of patients with $A B C$ vary among countries and are still not part of the clinical routine even in most countries with access to PARPi [27]. This survey evaluated awareness, clinical practice, and availability of routine diagnostic gBRCA1/2 mutation testing in the outpatient oncology setting in Germany, particularly for patients with HER2- ABC.

\section{Materials and Methods}

A multicentric, 23-item multiple-choice online survey (www. limesurvey.org) was completed by office-based oncologists across Germany from October 23, 2019 to February 3, 2020. Only oncologists treating patients with $\mathrm{ABC}$ were eligible. Clinicians practicing in hospitals, clinics, and direct members of the German Consortium for Hereditary Breast and Ovarian Cancer [28] were excluded. The German Consortium for Hereditary Breast and Ovarian Cancer, which currently consists of more than 20 university centers with multiple cooperation partners, was established to provide structured interdisciplinary care of individuals with a family history of breast and ovarian cancer in Germany [28].
Eligibility screening was conducted based on described criteria. All data were anonymized and analyzed descriptively as a whole and stratified by center type.

\section{Results}

\section{Participant Demographics}

Of 173 practices contacted, 53 agreed to participate, 21 declined, and 99 did not respond. Of the 53 practices who agreed to participate, 50 office-based oncologists completed the survey; around two-thirds were medical oncologists $(n=33)$ and one-third gynecological oncologists $(n=17)$. To our knowledge, this reflects the approximate proportion of medical and gynecological oncologists treating patients with $\mathrm{ABC}$ in an outpatient oncology setting in Germany. Most practices $(n=36)$ were associated with a breast cancer center certified by the German Cancer Society (Deutsche Krebsgesellschaft e.V.) and 5 were cooperation partners of the German Consortium for Hereditary Breast and Ovarian Cancer. Oncologists treated a median of 65 patients with $\mathrm{ABC}$ per year (range 14-350); this was consistent across medical and gynecological oncologists.

gBRCA1/2 Testing Decision Factors and Testing Rates

A known family history of gBRCA1/2 mutation-related cancer(s) (defined by the Arbeitsgemeinschaft Gynäkologische Onkologie e.V. [AGO] 2020 recommendations [23]), guideline recommendations, and triplenegative breast cancer (TNBC) were the strongest factors influencing an oncologist's decision to initiate gBRCA1/2 mutation testing (Fig. 1). Health economic factors such as therapy/test costs, and a patient's insurance type, as well as clinicopathological factors such as localization of metastases, a patient's general condition, histological grade, or medullary morphology, were considered but did not play a leading role in decision making.

In a routine outpatient oncology setting, $86 \%$ of oncologists reported testing patients with $\mathrm{ABC}$ for a gBRCA1/2 mutation. The selection of patients for mutation testing was strongly dependent on a known familial history of $\mathrm{gBRCA} 1 / 2$ mutation-related cancer(s) and type of breast cancer. In patients with a known familial history, testing was conducted in $98 \%, 82 \%, 82 \%$, and $76 \%$ of patients with advanced TNBC, HR+/HER2$\mathrm{ABC}, \mathrm{HR}-/ \mathrm{HER} 2+\mathrm{ABC}$, and HR+/HER2+ ABC, respectively. Notably, if the predisposition of a patient's family to a $g B R C A 1 / 2$ mutation-related cancer was unknown, only $30 \%$ of oncologists routinely tested patients with $\mathrm{HR}+/ \mathrm{HER} 2-\mathrm{ABC}$. In the absence of a known familial history, 92\% of oncologists reported routinely testing patients with TNBC (Fig. 2). 


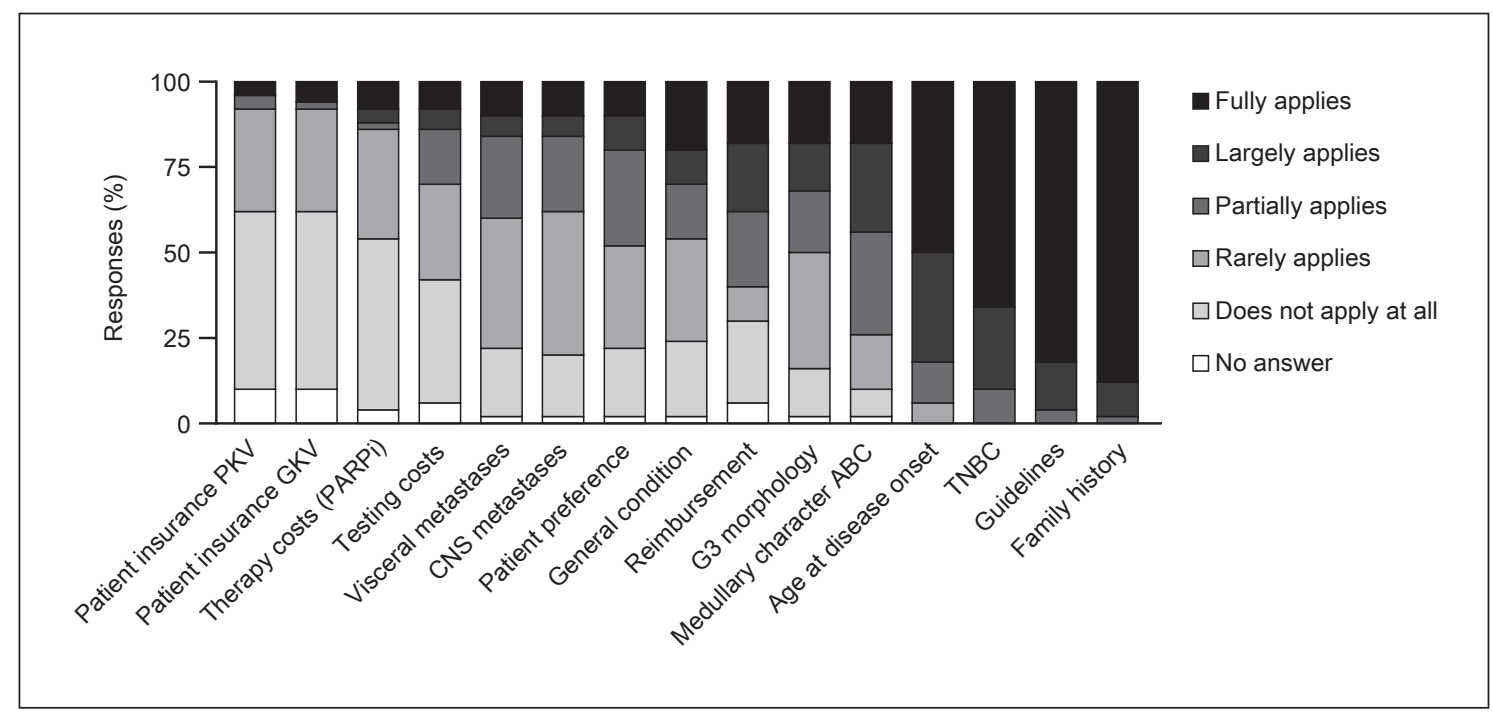

Fig. 1. Factors influencing an oncologist's decision to perform germline $B R C A 1 / 2$ testing for patients with $A B C$ $(N=50)$. ABC, advanced breast cancer; CNS, central nervous system; G, grade; GKV, statutory health insurance; PARPi, poly(ADP-ribose) polymerase inhibitor; PKV, private health insurance; TNBC, triple-negative breast cancer.

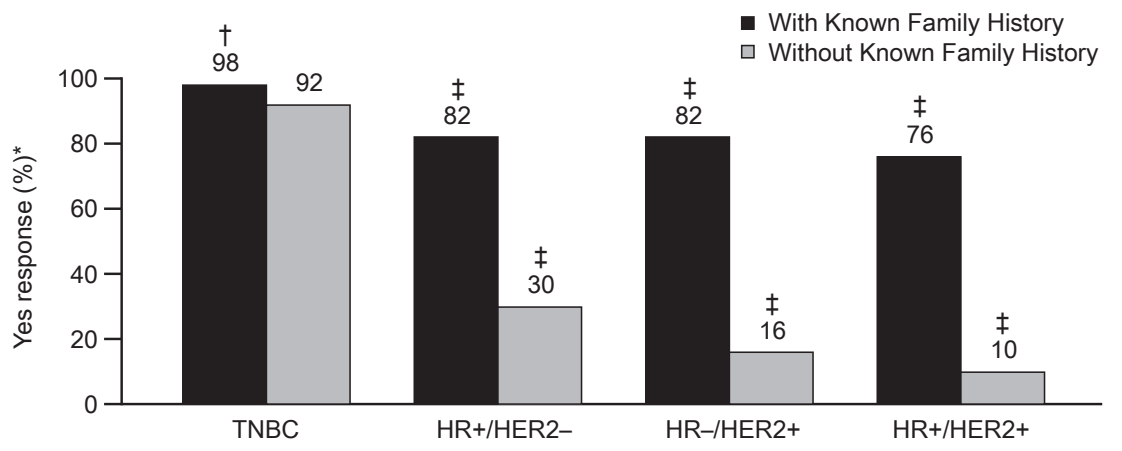

Fig. 2. Germline $B R C A 1 / 2$ testing rates according to receptor status and known family history of germline $B R C A 1 / 2$ mutation-related cancers for patients with $\mathrm{ABC}(N=50) .{ }^{*}$ The proportion of physicians who answered "Yes" to questions relating to which patients with $\mathrm{ABC}$ they would usually test for $\mathrm{g} B R C A 1 / 2$ mutation; percentages are based on the full survey population $(N=50) .{ }^{\dagger}$ One oncologist did not answer. ${ }^{\ddagger} 2$ oncologists did not answer. Reasons provided for not testing for $\mathrm{gBRCA1/2}$ in patients with a known family history were $(n=7)$ : in HR+/HER2- breast cancer patients, the availability of other treatment options $(n=7 ; 100 \%)$ and reim- bursement difficulties ( $n=2 ; 29 \%$ ). Reasons provided for not testing for $\mathrm{g} B R C A 1 / 2$ in patients without a known family history were $(n=33)$ : in HR+/HER2- breast cancer patients, the availability of other treatment options $(n=18 ; 55 \%)$, reimbursement difficulties $(n=8 ; 24 \%)$, and other reasons $(n=8 ; 24 \% ; 3$ oncologists did not answer); in patients with TNBC $(n=4)$, reimbursement difficulties ( $n=3 ; 75 \% ; 1$ oncologist did not answer). ABC, advanced breast cancer; gBRCA1/2, germline breast cancer susceptibility genes 1 or 2; HER2, human epidermal growth factor receptor 2; HR, hormone receptor; TNBC, triple negative breast cancer.

\section{Routine gBRCA1/2 Mutation Testing}

In an outpatient cancer setting, gBRCA1/2 mutation testing was carried out by various facilities. While $68 \%$ of surveyed oncologists confirmed their laboratory is accredited for $\mathrm{gBRCA} 1 / 2$ testing, $30 \%$ were not aware of the accreditation status of their testing facility; 1 oncologist (2\%) did not answer. No physician ordered testing from

a facility known to be non-accredited. Time from the initiation of testing to receipt of results ranged from 1 to 21 weeks with a median of 4 weeks; only $24 \%$ of oncologists received a test result within 2 weeks. This was comparable with sites that cooperated with the German Consortium for Hereditary Breast and Ovarian Cancer $(n=5$; median of 4 weeks; range 3-8 weeks). 


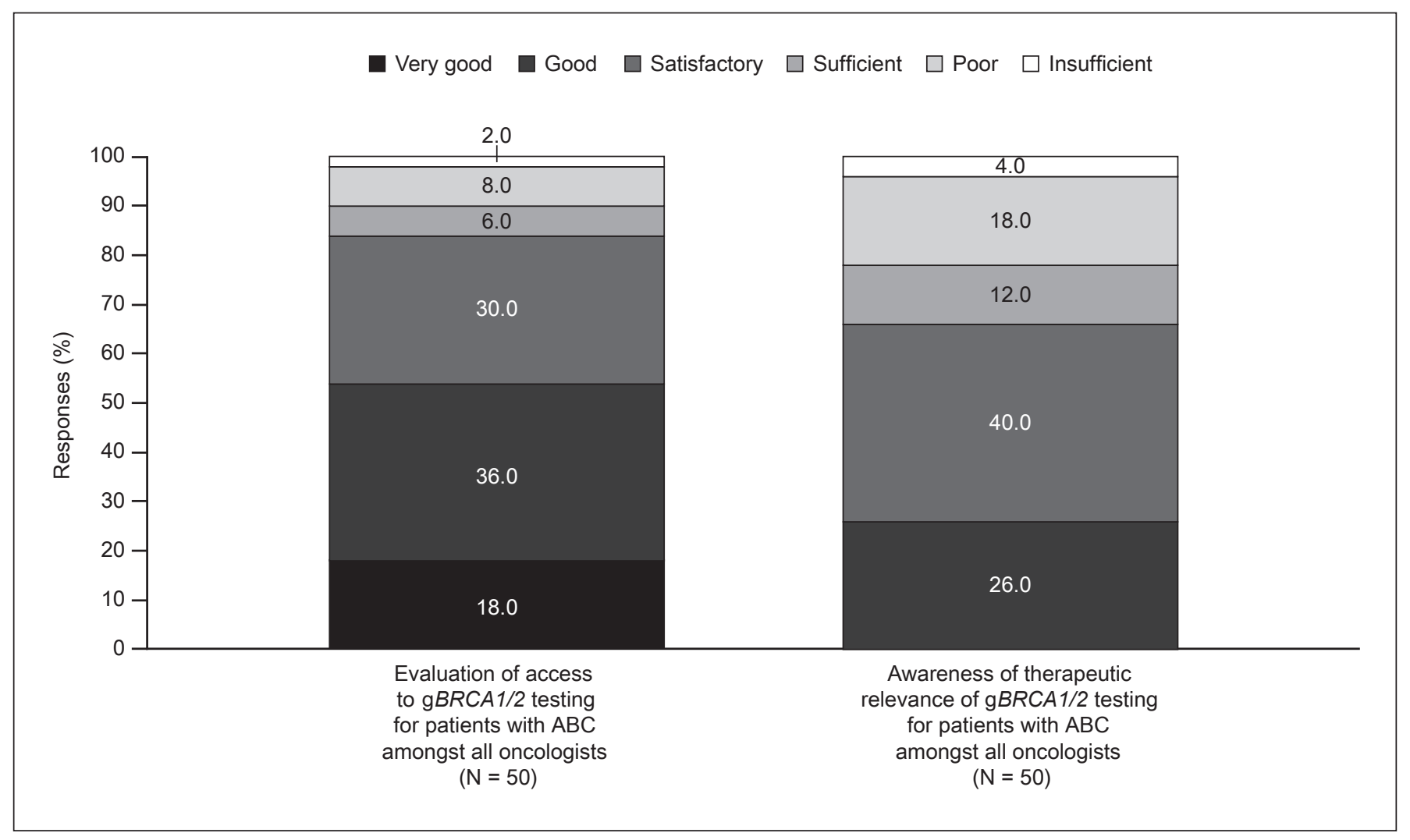

Fig. 3. Oncologists' evaluation of access to germline $B R C A 1 / 2$ testing for patients with $A B C$ and awareness of therapeutic relevance of germline $B R C A 1 / 2$ testing for patients with $\mathrm{ABC}$ (numbers may not add to 100 due to rounding). $\mathrm{ABC}$, advanced breast cancer; gBRCA1/2, germline breast cancer susceptibility genes 1 or 2 .

Given the therapeutic relevance of $\mathrm{gBRCA1/2}$ mutations for patients with HER2- ABC, genetic counseling is neither required to initiate $\mathrm{g} B R C A 1 / 2$ testing for these patients, nor to initiate PARPi therapy. However, oncologists must offer genetic counseling to patients where a (potentially) deleterious gBRCA1/2 mutation has been detected (GenDG \$10). In this survey, $94 \%$ of oncologists routinely advised patients to receive genetic counseling if a gBRCA1/2 mutation was detected. Most commonly, oncologists referred patients with a positive gBRCA1/2 mutation test result for genetic counseling to the German Consortium for Hereditary Breast and Ovarian Cancer (44\%); however, $28 \%$ of oncologists surveyed had qualified for genetic counseling according to the German Genetic Diagnostics Act (GenDG \$7) and provided counseling to their patients. For those oncologists who did not collaborate with the German Consortium for Hereditary Breast and Ovarian Cancer $(n=43 ; 86 \%), 93 \%$ informed patients about the option of genetic counseling if a (potentially) deleterious $\mathrm{g} B R C A 1 / 2$ mutation was detected. If the patient opted to receive genetic counseling, most oncologists referred their patient to an associated human geneticist (60\%) and/or counselled the patient themselves (42\%).
Approximately one-third (30\%) of oncologists received notification from their pathologists about potential familial breast cancer if the tumor tissue displayed typical histopathological features of BRCA1-associated breast cancer (invasive mammary carcinoma with medullary features and grade 3 morphology) [29]. Of the 15 oncologists who received this information, $73 \%$ routinely initiated testing for $\mathrm{g} B R C A 1 / 2$ mutations.

\section{Awareness and Access to gBRCA1/2 Testing}

General access to gBRCA1/2 testing in clinical practice was rated as very good or good by $54 \%$ of oncologists, while $10 \%$ rated access as poor or insufficient. Access was perceived as slightly better among medical oncologists (61\% rated very good or good) than gynecological oncologists ( $41 \%$ rated very good or good; Fig. 3). All 5 centers cooperating with the German Consortium for Hereditary Breast and Ovarian Cancer perceived access to $\mathrm{g} B R C A 1 / 2$ mutation testing as at least sufficient.

A total of $66 \%$ of oncologists rated awareness among oncologists of the therapeutic relevance of $\mathrm{g} B R C A 1 / 2$ mutation testing to identify patients with $A B C$ eligible for treatment with a PARPi as good or satisfactory. Notably, $22 \%$ of oncologists rated awareness as poor or insufficient 


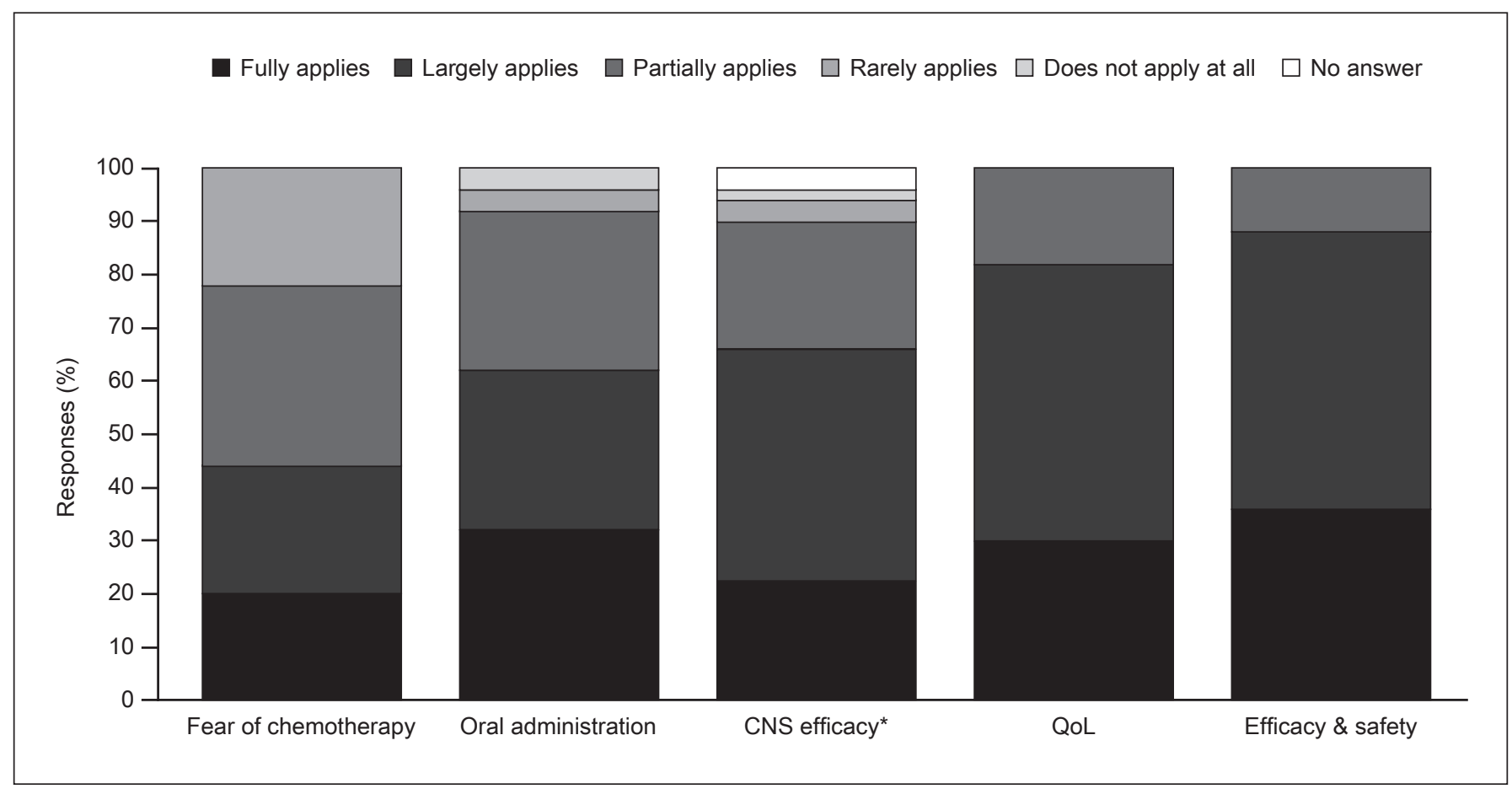

Fig. 4. Oncologists' opinions on the advantages of PARPi over conventional chemotherapy. * In the EMBRACA study, efficacy in terms of progression-free survival and tumor response was shown for patients with a known history of CNS metastasis [15, 19]. CNS, central nervous system; PARPi, poly(ADP-ribose) polymerase inhibitors; QoL, quality of life.

(Fig. 3). Only $6 \%$ of oncologists rated awareness of the therapeutic relevance of $\mathrm{g} B R C A 1 / 2$ mutation testing among patients as very good or good; most oncologists considered awareness as poor $(26 \%)$, sufficient $(20 \%)$, or satisfactory (34\%).

\section{PARPi and Chemotherapy}

When asked about the potential advantages of PARPi over chemotherapy, oncologists considered the following partially, largely, or fully applicable: proven efficacy and safety profile (100\%), patient's quality of life $(100 \%)$, flexibility of oral administration (92\%), efficacy in patients with a history of central nervous system metastasis (90\%), and fear of chemotherapy among patients (78\%; Fig. 4).

\section{Discussion}

The results from this survey describe the real-world practice of gBRCA1/2 mutation testing among community oncologists across Germany. Participating oncologists represented a broad range of office-based medical and gynecological oncologists who treated 14-350 patients with $A B C$ per year.

Diagnostic gBRCA1/2 mutation testing of patients with $\mathrm{ABC}$ was routinely performed in Germany's outpatient oncology setting. This aligns with previous reports, which indicated higher testing rates in countries with access to treatment with PARPi [27]. However, diagnostic gBRCA1/2 mutation testing was carried out with greatest frequency when a familial predisposition to $\mathrm{g} B R C A 1 / 2$ mutation-related cancer(s) was known, and/or when a patient presented with advanced TNBC. While this aligns with reported factors that influence an oncologist's decision to initiate gBRCA1/2 mutation testing in this survey, it does not fully align with current guideline recommendations $[23,25]$. This is notable as only one-third of oncologists reported routinely testing patients with $\mathrm{HR}+1$ HER2- ABC without a known familial predisposition, despite PARPi being effective alternatives to chemotherapy in patients with $\mathrm{HR}+$ disease $[15,16]$. Of note, about 1 in 20 patients with HR+/HER2- breast cancer have a gBRCA1/2 mutation regardless of a known family history $[2,30]$. gBRCA1/2 mutations are more frequent in patients with TNBC than those with HR+ disease [1]. However, since HR+ breast cancer accounts for approximately $80 \%$ of all breast cancers [31], increasing gBRCA testing in this population is an important and clinically meaningful consideration.

One quarter of oncologists who do not routinely test patients with HR+/HER2- ABC confirmed reimbursement difficulties as a contributing cause. Before January 2020 in Germany, there was no health insurance billing code number available for patients to undergo diagnostic 
gBRCA1/2 testing in the absence of an identified familial risk. Now, inclusion of a new doctor's fee scale also facilitates the reimbursement of diagnostic $\mathrm{g} B R C A 1 / 2$ testing in patients with HER2 - ABC without a known familial risk [32]. This may increase therapeutically relevant diagnostic $\mathrm{g} B R C A 1 / 2$ mutation testing of all patients with advanced HER2- breast cancer in Germany.

While the majority of physicians rated awareness of the therapeutic relevance of $\mathrm{g} B R C A 1 / 2$ mutation testing among oncologists as good or satisfactory, actual testing rates were low in certain subgroups, for example patients with $\mathrm{HR}+\mathrm{ABC}$ without a known familial predisposition. Thus, there is a need to increase oncologists' awareness of the therapeutic relevance of $\mathrm{g} B R C A 1 / 2$ mutation testing, similar to other European countries [27]. Three pillars are essential to increase awareness of the diagnostic relevance of $g B R C A 1 / 2$ testing. First, it is important to ensure and maintain awareness among treating physicians; this includes comprehensive sector-wide educational and training programs delivered by academia and medical societies, as well as guideline committees and industry. In today's digital age, access to advanced training should also remain available virtually. Second, it is necessary to closely collaborate with patient advocacy groups and increase patient awareness; this includes patient-orientated educational materials and lay language presentations of scientific research findings. Third, it is important to effectively plan the country-wide dissemination of research findings and educational programs, support local activities, and thus eventually reach a country-wide awareness of the therapeutic relevance of $\mathrm{g} B R C A 1 / 2$ testing.

Thus, in the age of precision medicine, broad, fast, and informed access to diagnostic testing is of paramount importance to guide treatment decisions. In Germany, gBRCA $1 / 2$ mutation testing in patients with HER $2-\mathrm{ABC}$ is considered routinely available and feasible in the outpatient oncology setting across center types. However, there is a need to improve therapeutically relevant $\mathrm{gBRCA} 1 / 2$ mutation testing. This holds true especially for patients with $\mathrm{HR}+/ \mathrm{HER} 2-\mathrm{ABC}$ without a known family history of $\mathrm{g} B R C A 1 / 2$ mutation-related cancer(s) to evaluate eligibility for targeted treatment with a PARPi.

\section{Acknowledgements}

Analyses and preparation of the final report was conducted by Ralf Zerbes and Stefan Zacharias of iOMEDICO, Freiburg, Germany. Antonin Kayser of Pfizer Germany critically reviewed the final report and Esther Glastetter of Pfizer Germany contributed to data interpretation. Melanie Frank of iOMEDICO, Freiburg, Germany, reviewed the manuscript to ensure correct statistical interpretation of the data. Medical writing support was provided by Zaavan Baildon of CMC AFFINITY, McCann Health Medical Communications, and funded by Pfizer. Final approval rested solely with the authors.

\section{Statement of Ethics}

As no patients were involved in this survey, no ethical approval was required.

\section{Conflict of Interest Statement}

M.P.L. reports honoraria for lectures, consulting, or an advisory role for Eli Lilly, AstraZeneca, MSD, Novartis, Pfizer, Eisai, Genomic Health, Roche, Hexal, and Medac; travel, accommodations, expenses from Roche and Pfizer; editorial board member of Medac; fees for non-CME services from Eli Lilly, Roche, Novartis, Pfizer, Genomic Health, AstraZeneca, MSD, and Eisai. T.D. reports advisory/consultancy fees for Novartis and Eli Lilly. E.D.R. and A.N. are employees of Pfizer and report ownership interest in Pfizer. R.G.W.Q. was an employee of Pfizer at the time of the analyses and reported ownership interest in Pfizer and Amgen. N.M. reports ownership and a leading position within iOMEDICO, and research grants from Pfizer in the last 3 years. N.H. reports honoraria from Pfizer, AstraZeneca, and MSD.

\section{Funding Sources}

This survey was sponsored by Pfizer Pharma GmbH. Medical writing was sponsored by Pfizer Inc.

\section{Author Contributions}

The authors had full access to the data and take responsibility for the integrity of the data and accuracy of the analysis. E.D.R., A.N., and R.G.W.Q.: survey concept and design. N.M.: acquisition of data and statistical analysis (iOMEDICO AG, Freiburg, Germany). M.P.L., T.D., E.D.R., A.N., R.G.W.Q., N.M., and N.H.: analysis and interpretation of data. E.D.R.: drafting of the manuscript. M.P.L., T.D., A.N., R.G.W.Q., N.M., and N.H.: critical revision of the manuscript for important intellectual content. E.D.R.: obtaining funding.

\section{Availability of Data}

Data are available for bona fide researchers who request it from the authors.

\section{References}

1 Armstrong N, Ryder S, Forbes C, Ross J, Quek RG. A systematic review of the international prevalence of BRCA mutation in breast cancer. Clin Epidemiol. 2019 Jul;11:543-61.

2 Tung N, Lin NU, Kidd J, Allen BA, Singh N, Wenstrup RJ, et al. Frequency of germline mutations in 25 cancer susceptibility genes in a sequential series of patients with breast cancer. J Clin Oncol. 2016 May;34(13):1460-8.

3 Kast K, Rhiem K, Wappenschmidt B, Hahnen E, Hauke J, Bluemcke B, et al.; German Consortium for Hereditary Breast and Ovarian Cancer (GC-HBOC). Prevalence of BRCA1/2 germline mutations in 21401 families with breast and ovarian cancer. J Med Genet. 2016 Jul;53(7):465-71. 
4 Kang E, Seong MW, Park SK, Lee JW, Lee J, Kim LS, et al.; Korean Hereditary Breast Cancer Study Group. The prevalence and spectrum of BRCA1 and BRCA2 mutations in Korean population: recent update of the Korean Hereditary Breast Cancer (KOHBRA) study. Breast Cancer Res Treat. 2015 May;151(1): 157-68.

5 Kuchenbaecker KB, Hopper JL, Barnes DR, Phillips KA, Mooij TM, Roos-Blom MJ, et al. Risks of breast, ovarian, and contralateral breast cancer for BRCA1 and BRCA 2 mutation carriers. JAMA. 2017 Jun;317(23):240216.

6 National Cancer Institute. BRCA Mutations: Cancer Risk and Genetic Testing. Available from: https://www.cancer.gov/about-cancer/ causes-prevention/genetics/brca-factsheet\#q1. Accessed June 24, 2020

7 Kim R, Peterson A, Isherwood A, Uppal H, Barlev A. Incidence of germline BRCA1- and BRCA2-mutated breast cancer in the US [abstract]. Cancer Res. 2017;77(4 Suppl):08-28.

8 National Comprehensive Cancer Network (NCCN). NCCN Clinical Practice Guidelines in Oncology (NCCN Guidelines ${ }^{\circledR}$ ): Genetic/ familial high-risk assessment: breast, ovarian, and pancreatic. Version 1. 2020. Available from: https://www.nccn.org/professionals/ physician_gls/pdf/genetics_bop.pdf. Accessed July 1, 2020.

9 U.S. Food and Drug Administration. TALZENNA $^{\circledR}$ (talazoparib) prescribing information. 2020. Available from: http://labeling. pfizer.com/ShowLabeling.aspx?id=11046. Accessed October 08, 2020.

10 U.S. Food and Drug Administration. LYN PARZA $^{\circledR}$ (olaparib) prescribing information. 2020. Available from: https://www.azpicentral.com/lynparza_tb/lynparza_tb. pdf\#page=1. Accessed August 24, 2020.

11 European Medicines Agency. TALZENNA ${ }^{\circledR}$ (talazoparib) Summary of Product Characteristics. 2019. Available from: https://www. ema.europa.eu/en/documents/product-information/talzenna-epar-product-information_en.pdf. Accessed October 08, 2020.

12 European Medicines Agency. LYNPARZA ${ }^{\circledR}$ (olaparib) Summary of Product Characteristics. 2019. Available from: https://www.medicines.org.uk/emc/product/9204/smpc. Accessed June 26, 2020.

13 Gemeinsamer Bundesausschuss. Benefit assessment procedure for olaparib. 2020. Available from: https://www.g-ba.de/bewertungsverfahren/nutzenbewertung/463/\#dossier. Accessed October 30, 2020.

14 Gemeinsamer Bundesausschuss. Benefit assessment procedure for talazoparib. 2020.
Available from: https://www.g-ba.de/bewertungsverfahren/nutzenbewertung/554/\#stell ungnahmen. Accessed October 30, 2020.

15 Litton JK, Rugo HS, Ettl J, Hurvitz SA, Gonçalves A, Lee KH, et al. Talazoparib in patients with advanced breast cancer and a germline BRCA mutation. N Engl J Med. 2018 Aug; 379(8):753-63.

16 Robson M, Im SA, Senkus E, Xu B, Domchek SM, Masuda N, et al. Olaparib for Metastatic Breast Cancer in Patients with a Germline BRCA Mutation. N Engl J Med. 2017 Aug; 377(6):523-33.

17 Litton JK, Hurvitz SA, Mina LA, Rugo HS, Lee $\mathrm{KH}$, Gonçalves A, et al. Talazoparib versus chemotherapy in patients with germline BRCA1/2-mutated HER2-negative advanced breast cancer: final overall survival results from the EMBRACA trial. Ann Oncol. 2020 Nov;31(11):1526-35.

18 Robson ME, Tung N, Conte P, Im SA, Senkus $\mathrm{E}, \mathrm{Xu} \mathrm{B}$, et al. OlympiAD final overall survival and tolerability results: olaparib versus chemotherapy treatment of physician's choice in patients with a germline BRCA mutation and HER2-negative metastatic breast cancer. Ann Oncol. 2019 Apr;30(4):558-66.

19 Rugo HS, Ettl J, Hurvitz SA, Gonçalves A, Lee $\mathrm{KH}$, Fehrenbacher L, et al. Outcomes in Clinically Relevant Patient Subgroups From the EMBRACA Study: Talazoparib vs Physician's Choice Standard-of-Care Chemotherapy. JNCI Cancer Spectr. 2019 Oct;4(1):pkz085.

20 Ettl J, Quek RG, Lee KH, Rugo HS, Hurvitz S, Gonçalves A, et al. Quality of life with talazoparib versus physician's choice of chemotherapy in patients with advanced breast cancer and germline BRCA $1 / 2$ mutation: patientreported outcomes from the EMBRACA phase III trial. Ann Oncol. 2018 Sep;29(9): 1939-47.

21 Hurvitz SA, Gonçalves A, Rugo HS, Lee KH, Fehrenbacher L, Mina LA, et al. Talazoparib in Patients with a Germline BRCA-Mutated Advanced Breast Cancer: Detailed Safety Analyses from the Phase III EMBRACA Trial. Oncologist. 2020 Mar;25(3):e439-50.

22 Robson M, Ruddy KJ, Im SA, Senkus E, Xu B, Domchek SM, et al. Patient-reported outcomes in patients with a germline BRCA mutation and HER2-negative metastatic breast cancer receiving olaparib versus chemotherapy in the OlympiAD trial. Eur J Cancer. 2019 Oct;120:20-30.

23 Kommission Mamma der Arbeitsgemeinschaft Gynäkologische Onkologie V. (AGO) [AGO Breast Committee]. Diagnostik und Therapie früher und fortgeschrittener Mammakarzinome [Diagnosis and Treatment of
Patients with Primary and Metastatic Breast Cancer]. Version 1. 2020. Available from: https://www.ago-online.de/fileadmin/agoonline/downloads/_leitlinien/kommission mamma/2020/Alle_aktuellen_Empfehlungen_2020.pdf. Accessed June 24, 2020.

24 National Comprehensive Cancer Network (NCCN). NCCN Clinical Practice Guidelines in Oncology (NCCN Guidelines ${ }^{\circledR}$ ): Breast Cancer. Version 5. 2020. Available from: https://www.nccn.org/professionals/physician_gls/pdf/breast_blocks.pdf. Accessed October $08,2020$.

25 Thomssen C, Lüftner D, Untch M, Haidinger R, Würstlein R, Harbeck N, et al. International Consensus Conference for Advanced Breast Cancer, Lisbon 2019: ABC5 Consensus - Assessment by a German Group of Experts [Conference Report]. Breast Care. 2020 Feb; 15(1):82-95.

26 Tung NM, Boughey JC, Pierce LJ, Robson ME, Bedrosian I, Dietz JR, et al. Management of Hereditary Breast Cancer: American Society of Clinical Oncology, American Society for Radiation Oncology, and Society of Surgical Oncology Guideline. J Clin Oncol. 2020 Jun;38(18):2080-106.

27 Mahtani RL, Niyazov A, Lewis K, Wild R, Rider A, Arondekar B, et al. Germline BRCA1/2 (GBRCA1/2) Testing Patterns Among Oncologists (ONC) Treating HER2- Advanced Breast Cancer (ABC): Results From A MultiCountry Real-World Study [abstract]. Ann Oncol. 2020;31(Suppl 2):S62-82.

28 Deutsches Konsortium Familiärer Brust- und Eierstockkrebs [German Consortium of Familial Breast and Ovarian Cancer]. Available from: https://www.konsortium-familiaererbrustkrebs.de. Accessed November 6, 2020.

29 Honrado E, Benítez J, Palacios J. The molecular pathology of hereditary breast cancer: genetic testing and therapeutic implications. Mod Pathol. 2005 Oct;18(10):1305-20.

30 Fasching PA, Hu C, Hart SN, Polley EC, Lee $\mathrm{KY}$, Gnanolivu RD, et al. Cancer predisposition genes in metastatic breast cancer - Association with metastatic pattern, prognosis, patient and tumor characteristics [abstract]. Cancer Res. 2018;78(4 Supplement):PD1-02.

31 Howlader N, Altekruse SF, Li CI, Chen VW, Clarke CA, Ries LA, et al. US incidence of breast cancer subtypes defined by joint hormone receptor and HER2 status. J Natl Cancer Inst. 2014 Apr;106(5):dju055. https://doi. org/10.1093/jnci/dju055.

32 Kassenärztliche Bundesvereinigung. Einheitlicher Bewertungsmassstab (EBM). 2020. Available from: https://www.kbv.de/html/ ebm.php. Accessed July 7, 2020. 